\title{
INVESTIGACIÓN
}

Recibido: 12/03/2021 --- Aceptado: 15/04/2021 --- Publicado: 24/05/2021

\section{SUPERMERCADOS, REDES SOCIALES Y COVID-19: NEUROMARKETING Y HUMANIZACIÓN DEL MENSAJE}

\section{Supermarkets, social media and covid-19: neuromarketing and humanisation of the message}

Patricia Ardanza-Ruiz. Cesine. España.

patriciaardanza@ardanza.com

(D) $\mathrm{R}^{\mathrm{T}}$ José M. Lavín. Cesine. España.

jm.lavin@cesine.com

\section{Como citar el artículo:}

Ardanza-Ruiz, A. y Lavin, J. M. (2021). Supermercados, redes sociales y covid-19: neuromarketing y humanización del mensaje.Vivat Academia. Revista de Comunicación, 154, 361-379. http:// doi.org/10.15178/va.2021.154.e1356

\section{RESUMEN}

Los supermercados utilizan toda serie de técnicas de marketing para atraer a sus clientes potenciales a su establecimiento e incitarles a comprar sus productos. Una de las técnicas más novedosas en este ámbito ha sido el neuromarketing, ciencia que estudia los efectos de la publicidad y otras acciones de comunicación en el cerebro humano con la intención de poder llegar a predecir la conducta del consumidor a partir de las impresiones creadas. Este trabajo se centra en el estudio de las técnicas de neuromarketing utilizadas por los supermercados de nuestro país en sus redes sociales durante el confinamiento debido a la pandemia de la COVID-19, de forma que se pueda determinar que dichas técnicas varían en comparación de situaciones normales, para lo que se han utilizado como ejemplos Carrefour e Hipercor. Se ha realizado una investigación con enfoque cualitativo donde se ha desarrollado una comparativa sobre la comunicación en redes sociales (Instagram, Facebook y Twitter) realizada por dichos supermercados a nivel nacional antes de la crisis de la COVID19 y durante la misma. Se ha observado que la publicidad varió de un $10-30 \%$ de comunicación enfocada al cliente antes de la crisis, a un 100\% durante la misma. Llegando a la conclusión de que en tiempo de crisis el neuromarketing sale más a la luz, humanizando más a las empresas para situarse más cerca del cliente. También se puede observar que las técnicas usadas varían dependiendo de cada supermercado y de la etapa de la crisis por la que esté pasando. 
PALABRAS CLAVE: Neuromarketing en supermercados - Neuromarketing durante el Covid19 - publicidad dirigida al cliente - cambio de estrategia en publicidad cambio de estrategia en marketing - Marketing y pandemia - Publicidad y pandemia.

\section{ABSTRACT}

Supermarkets use a variety of marketing techniques to attract potential customers to their shops and encourage them to buy their products. One of the newest techniques in this field has been neuromarketing, a science that studies the effects of advertising and other communication actions on the human brain with the intention of being able to predict consumer behaviour. This document focuses on the study of the neuromarketing techniques used by supermarkets in our country in their social networks during the confinement due to the COVID-19 pandemic, so that it can be determined that these techniques vary in comparison with normal situations, for which Carrefour and Hipercor have been used as examples. A qualitative research study was carried out to compare the communication on social networks (Instagram, Facebook and Twitter) carried out by these supermarkets at national level before and during the COVID-19 crisis. It was observed that advertising varied from 10-30\% customer-focused communication before the crisis to $100 \%$ during the crisis. This leads to the conclusion that in times of crisis neuromarketing comes to the fore, humanising companies more to get closer to the customer. It can also be observed that the techniques used vary depending on each supermarket and the stage of the crisis they are going through.

KEYWORDS: Neuromarketing in supermarkets - Neuromarketing during the COVID-19 - advertising aimed at the client - change of strategy in advertising change of strategy in marketing - Marketing and pandemic - Advertising and pandemic.

\section{INTRODUCCIÓN}

El término neuromarketing como se conoce hoy en día comenzó a utilizarse en 2002 por Ale Smidts, pero se puede comenzar a hablar de él como ciencia desde 1881, momento en el que apareció la neurociencia como tal, gracias a un experimento de Angelo Mosso, científico italiano (Ciprian-Marcel et al, 2009:804). En este experimento, Mosso se dio cuenta de que la pulsación de las personas variaba durante la fase mental, y de que la actividad emocional afectaba al flujo sanguíneo y la redistribución de la sangre por el cuerpo, aumentando las pulsaciones del individuo cuando se producía cierta actividad emocional. Así, se concluyó que, si se estudiaban dichas reacciones a las emociones en los diferentes usuarios, se podría obtener información de gran utilidad acerca del comportamiento humano. Y esto es exactamente lo que hace el neuromarketing al exponer a los clientes ante la publicidad, las marcas y los productos.

Ahora bien, existen diferentes definiciones sobre el neuromarketing. Según Kotler y Armstrong (2012), se puede definir como una ciencia que estudia detalladamente 
las respuestas de los clientes y consumidores de una empresa ante un estímulo concreto, para lo que utiliza técnicas de la neurociencia como el electroencefalograma o la resonancia magnética. También puede definirse como la manera científica de demostrar por qué las personas reaccionamos de una manera $u$ otra ante los estímulos de compra, por lo que ayuda a las empresas en la creación de valor y en su propio crecimiento, ya que les permite identificar necesidades de los clientes que no han sido satisfechas anteriormente dándoles la opción de cubrirlas (Wind, 2004). Destacar también la definición aportada por Klaric (2011) en la que dice que el neuromarketing es el estudio de los efectos de la publicidad y otras acciones de comunicación en el cerebro humano con la intención de poder llegar a predecir la conducta del consumidor, conectando de manera estratégica los productos, servicios, marcas o categorías con la mente del cliente.

Se suele hacer diferencias entre el neuromarketing visual, el auditivo y el kinestésico, que engloba el tacto, el gusto y el olfato. Según Braidot (2005), cuando se reciben estímulos del exterior mediante los sentidos, el cerebro registra, procesa e interpreta dicha información. Así se puede ver que cada persona realizará este proceso obteniendo una información diferente, ya que no serán cautivados por los mismos estímulos. Si los estímulos proceden de diferentes sentidos, la información también variará. Por tanto, el marketing sensorial es esencial para conseguir atraer la atención de los consumidores.

Hay que añadir que, gracias al neuromarketing, se han hecho diferentes estudios en los que se ha determinado que el desarrollo de ciertas actividades por parte de la empresa, denominadas acciones de Responsabilidad Social Corporativa (RSC) (Lee, 2016) llegan de una mejor manera a la parte frontal del cerebro, parte que se ha comprobado que es donde nace la empatía de los individuos. Se puede interpretar entonces que, si las empresas consiguen llegar a esta parte del cerebro de los consumidores, éstos tendrán una mejor imagen de ellos y, por tanto, podrán ser influenciados en su proceso de compra. Finalmente, como bien concluye Eun-Ju Lee (2016:7) "Marketers can understand that consumers high in empathy trait will be less sensitive to price increases associated with corporates' social campaigns.", aspecto que se ha de tener en cuenta a la hora de desarrollar este tipo de campañas. Es por esto que algunos estudios relacionan el hecho de llevar a cabo acciones de RSC con la creación de actitudes positivas hacia los productos (Lee, 2016).

\subsection{Técnicas concretas de neuromarketing}

Cabe mencionar los principales aspectos que se tienen en cuenta durante la experiencia de compra: la información, la ambientación, la simplicidad y el entretenimiento (Pradeep, 2010). En cuanto a la información, se puede decir que cuando un individuo acude a un supermercado, tiene el suficiente conocimiento sobre los productos que puede o desea comprar. Se le debe aportar la suficiente información para encontrar los productos de manera sencilla, para lo que cabe destacar que, en los últimos años, se ha comprobado que es más efectivo el uso de imágenes e iconos porque tiene mayor efecto de recordación (Pradeep, 2010). La

Vivat Academia. Revista de Comunicación. 2021, n 154, 361-379 
ambientación hace referencia a la parte física de la tienda, por lo que juega un papel esencial a la hora de "agradar" a los consumidores y hacer que se motiven a comprar. Respecto a la simplicidad, el supermercado debe hacer la compra lo más sencilla posible para el consumidor, cuantos más impedimentos encuentre éste, más probabilidades habrá de que abandone la compra. Aquí se puede destacar el hecho de que un continuo rediseño del supermercado puede incordiar y ser perjudicial para los consumidores. Finalmente, se puede hablar de que a la gente le suele agradar el entretenimiento mientras compra, lo que puede hacer que alargue su tiempo en la misma (Pradeep, 2010).

A continuación, se muestran las principales y más comunes acciones llevadas a cabo en los supermercados gracias a la aplicación del neuromarketing:

\subsubsection{Eyetracking}

El estudio del eyetracking se centra en los movimientos del ojo con el fin de medir con precisión dónde se centra la atención de los consumidores durante la compra, ya sea compra física fijándose en las estanterías de los supermercados o compra online donde clica el ratón. Mediante este estudio también se puede estudiar la dilatación de las pupilas con el fin de determinar el grado de interés ante ciertos estímulos (Hess, 1975), así como el número de parpadeos que indican el grado emocional ante el mismo (Lang, 1995). Por tanto, ante este tipo de estudios, los expertos de marketing lo que deben preguntarse es qué es preferible para la compra, que sea una fijación más larga o corta, lo que les puede dar pistas, por ejemplo, de qué tipo de diseños de packaging deben aplicar a sus productos.

Por ejemplo, según Piqueras-Fiszman et al. (2013), la forma del envase y el etiquetado del mismo tienen una gran influencia en la decisión de compra. Lo mismo ocurrió en Koenig Storfer et al. (2013) donde se muestra que la forma en la que se muestra la información nutricional puede hacer que los individuos compren o no el producto.

Berč́k et al. (2015) mostró, gracias al eye tracker, que los consumidores suelen ser más receptivos ante estanterías llenas y bien ordenadas. El experimento se realizó con botellas de vino, y como se puede ver en la siguiente imagen, los puntos de calor se muestran mayoritariamente en la estantería llena. 


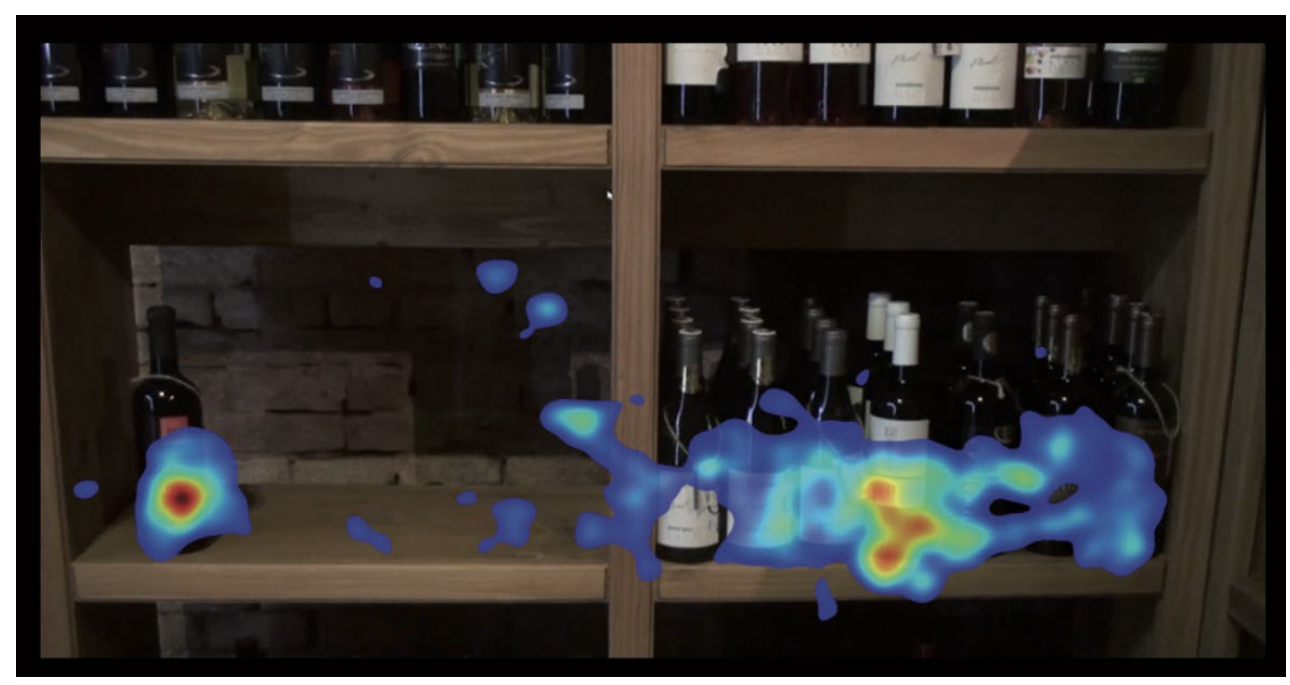

Figura 1: Eyetracking: Mapa de calor para experimento: estantería llena vs estantería vacía.

Fuente: Berč́k et al. (2015).

Además, en este mismo estudio se observó cómo el diseño del envase en las botellas de vino seguía un patrón único para llamar la atención de los consumidores. Así pues, llamaban más la atención las botellas oscuras con diseños en colores claros, sobre las que tenían un diseño más oscuro en general. Estudios similares a este han demostrado que los consumidores prefieren envases donde destaque el brillo y la intensidad de estos a la hora de comprarlos, de hecho, son factores que pueden hacer que un individuo elija dicho producto antes que otro que le guste más (Milosavljevic et al., 2011).

\subsubsection{Precio}

Finalmente, hay que mencionar el tema del precio como un elemento más utilizado por el neuromarketing para llamar la atención de los individuos en el proceso de compra. El precio marca en muchas ocasiones la diferencia, un individuo puede decidir si comprar o no un producto debido al precio. Plassmann et al. (2008) demostraron que, en muchas ocasiones, los individuos se guían del precio de los productos para determinar su calidad. Dicho estudio analizó el comportamiento de varios individuos ante una cata de vinos, para la cual dio a probar el mismo vino de diferentes botellas y diferentes precios. Así demostró que muchos individuos escogen la botella más cara por creer que tenía una calidad superior.

También se puede ver un ejemplo claro en el uso del 0,99€ para "confundir" al consumidor, hacerle ver un precio y que en su cabeza parezca menos de lo que realmente es.

\subsubsection{Responsabilidad Social Corporativa}


La Responsabilidad Social Corporativa (RSC) es una técnica más de lo que se conoce como neuromarketing, gracias a la cual se consiguen crear ciertas actitudes positivas o negativas respecto a ciertos productos. Es por esto por lo que diversos estudios de la neurociencia han demostrado que existen perfiles de consumidor únicos para aquellos individuos que compran productos relacionados con el cuidado del medioambiente, de la sociedad y de los animales, entre otros (Navarro García, 2012).

Un ejemplo claro de esto se puede ver en lo conocido como "Green marketing" (Peattie and Charter, 2003), que se ha puesto muy de moda en los últimos años. Este marketing se podría analizar desde su marketing mix de la siguiente manera:

En cuanto al "producto verde", las empresas están en continua búsqueda de productos nuevos que sean sostenibles y ecológicos, de forma que les pueda servir para atraer a nuevos consumidores concienciados con el medioambiente (Peattie and Charter, 2003). En ese aspecto se ha desarrollado lo conocido como "Design for Environment" que busca crear productos que encajen perfectamente con las necesidades del medioambiente, sin dañarlo, así como productos a los que se les pueda dar un uso después. Incluir también que el packaging y el etiquetado tienen también mucha importancia en esta parte, ya que el packaging es el primer aspecto que las empresas tienden a modificar para acercarse a ese "Green marketing" con el fin de darse un lavado de cara ante sus clientes, ya que no suele crear elevados costes. Y el etiquetado ya que ayuda al consumidor a saber posteriormente cómo utilizar el producto y cómo reciclarlo, dándole ese uso después de su vida útil.

En el tema de la promoción, lo que se busca es conseguir desarrollar "comunicaciones sostenibles" que se basan en informar y educar a los consumidores acerca del cuidado del entorno, desarrollando esa concienciación por el medioambiente y la sociedad.

En lo que se refiere al precio, las empresas están creando ese "precio verde", que hace referencia al aumento del precio sobre el producto por ser "green", ya que ello le ha generado a la empresa más costes (Peattie \& Charter, 2003).

\subsection{Neuromarketing en redes sociales}

En los últimos años, las relaciones entre empresas y consumidores se han visto influenciadas en gran medida por las redes sociales. Según un estudio realizado por Cervantes J., et al. (2016), gracias a este nuevo medio de comunicación las empresas consiguen crear una mayor interactividad con los clientes y hacen más propensas las ventas directas, ya que consiguen conectar con el cliente con una mayor rapidez y evitando en gran parte el desgaste con el resto de competencia. Hay que añadir que, gracias al uso de las redes sociales, el neuromarketing ha podido estudiar la forma de relacionarse de los consumidores en dichos medios, llegando a la conclusión de que existe una "influencia social" por la cual la gente tiende a imitar el comportamiento del resto. Es decir, cuando un usuario comparte la publicación de una marca, el resto 
tiende a imitarlo. Este es un aspecto para destacar en el caso de estudio con la COVID-19, ya que si las marcas consiguen que un usuario se conciencie con las medidas que ellos mismos comparten, el resto de los individuos le imitarán.

En relación a lo anterior, mencionar el estudio llevado a cabo por Muñoz-Leiva et al. (2018), en el que destaca que gracias a las redes sociales y al neuromarketing los expertos en comunicación han comenzado a redefinir su público objetivo, ya que les es más fácil saber qué estímulos afectan a cada individuo, así como los gustos que tiene, de tal forma que es más fácil "bombardearlos" con mensajes que les puedan llamar la atención, pero que al mismo tiempo pueden llegar a considerarse intrusivos dando lugar a percepciones negativas. Esto ha hecho que el concepto de "banner blindness" aumente, haciendo que los clicks en los mismos disminuyan.

Además, se puede destacar el estudio de Abuín-Vences., et al. (2020) en el que analizan diferentes investigaciones acerca del uso por parte de las empresas de las redes sociales en diferentes ámbitos. De este estudio se pueden destacar varias de sus conclusiones: en primer lugar, gracias al neuromarketing, los anunciantes están consiguiendo recopilar gran cantidad de datos que les son útiles para determinar la eficacia de los anuncios en las redes sociales; en segundo lugar, se ha analizado que el contenido que más repercusión tiene en los usuarios son los posts con contenido de entretenimiento seguidos de los que contienen información relacionada con la marca, donde destacan las fotografías como contenido preferido, y se rechazan los posts con gran contenido de texto, y finalmente que los posts con más comentarios son los publicados entre semana; además se ha comprobado que hay cierta relación entre los comentarios positivos en una publicación y su número de likes; gracias al neuromarketing se ha demostrado que es más efectiva la publicidad emocional que la puramente racional y que el uso de las redes sociales radica de la necesidad de los usuarios de satisfacer sus necesidades sociales y encontrar la aceptación del resto en sus decisiones; destacar que en tiempos de crisis los usuarios valoran positivamente que las marcas respondan a comentarios en sus redes; los post con mensajes emocionales (tanto positivos como negativos) tienden a generar mayor impacto y suelen presentar altos niveles de viralidad; finalmente destacar que, en este estudio se pudo observar que Facebook e Instagram se utilizan para generar post sobre emociones positivas, mientras que Twitter y Messenger generalmente se utilizan para lanzar post negativos.

Por otro lado, en un estudio de Aguirre et al. (2015), se demostró que cuando las empresas recogen información de los clientes para personalizar los mensajes de los anuncios online, éstos se pueden volver en su contra al sentir que se ha violado su privacidad. Este aspecto se estudió en un caso concreto en Facebook, donde se observó que los usuarios experimentaban fuertes sentimientos de vulnerabilidad.

También se puede mencionar el hecho de que, gracias a las redes sociales, las marcas crean vínculos con sus clientes. Cuando un usuario interacciona con una marca, ya sea a través de redes o participando en alguna acción creada por la marca, es muy fácil que éste cree vínculos con la marca (Matellanes Lazo, 2016). Además, se 
ha observado que se pueden llegar a crear 4 perfiles diferentes en función de dichos vínculos emocionales: el cliente transaccional, el cliente contento, el cliente leal y los fans. Es importante tener estos perfiles en cuenta a la hora de mejorar el engagement entre marca y cliente (Sashi, 2012).

Por otro lado, gracias a un estudio realizado por Muñoz-Leiva et al. (2018), se ha podido observar gracias a la técnica del eye-tracking cuáles son las partes de la pantalla que más miran los usuarios cuando navegan por las redes, lo que puede ayudar a las marcas a crear mensajes más efectivos. Una de las redes analizadas fue Facebook, donde se comprobó que los usuarios prestaban la mayor parte de su atención al header del post y posteriormente a los comentarios de otros consumidores.

Gracias al reciente estudio de Sano et al. (2019), donde se analizaron las reacciones de diferentes usuarios de Japón ante las redes sociales a lo largo de 10 años, se demostró que existen diferentes períodos que se repiten año tras año en los cuáles se repiten determinados estados de ánimo en los usuarios. Estos períodos suelen estar relacionados con fechas específicas como Navidad, Año Nuevo, el comienzo de las vacaciones, San Valentín, y a su vez, con catástrofes o fenómenos naturales que alteran la vida cotidiana.

También sería conveniente destacar el estudio llevado a cabo por Daniel Gómez (2014) en el cual se estudiaron las reacciones de varios usuarios ante diferentes campañas publicitarias llevadas a cabo en redes sociales. De dicha investigación se obtuvieron una serie de conclusiones: en primer lugar que en la mayoría de casos se activaba la parte de la corteza prefrontal del consumidor (lugar donde se ubica el llamado "Botón de compra), al ver el estímulo visual derivado de la campaña; que en la mayoría de casos los usuarios solo dan like a las páginas de empresas que encuentran llamativas, por lo que se deduce que éstas encuentran los estímulos adecuados para llamar la atención de los mismos; que las redes sociales son la herramientas definitiva para establecer las interacciones con los consumidores, donde pueden obtener información acerca de sus necesidades a través de los comentarios; y finalmente, que si las empresas utilizaran más las herramientas que ofrece el neuromarketing para analizar sus publicaciones en redes, conseguirían resultados más óptimos y evitarían que sus campañas fueran rechazadas por los consumidores.

\section{OBJETIVOS}

El objetivo general de este trabajo es conocer cómo Hipercor y Carrefour variaron sus estrategias de comunicación y el contenido de esta, incluyendo neuromarketing en redes sociales en vistas de la situación de España con la Covid-19.

1. Como objetivo específico número uno se analizará el cambio de la comunicación de Hipercor durante el periodo del confinamiento severo entre marzo y junio.

2. Como objetivo específico número dos se analizará el cambio de la comunicación de Carrefour durante el periodo del confinamiento severo entre marzo y junio. 
3. Como tercer objetivo específico se analizará el tipo de mensaje lanzado por ambas empresas que buscaba crear mayor empatía y cercanía con los clientes.

\section{METODOLOGÍA}

Para investigar dichos puntos, se va a realizar una investigación bajo un enfoque cualitativo. Se procederá a realizar una comparativa sobre la comunicación realizada por algunos supermercados nacionales antes del período de confinamiento severo en la crisis de la COVID-19 y durante la misma, con el fin de conocer y explicar las posibles diferencias y semejanzas de una situación a otra. Esta comparativa buscaría ver cuál fue el cambio de estrategia y de mensaje buscando empatía y convertirse en marcas amigables para la ciudadanía.

Los estudios comparativos constan de varias etapas (Gómez-Díaz de León y León de la Garza, 2015):

En la primera etapa, se ha de desarrollar un marco teórico sobre el que se pueda apoyar posteriormente la propia comparación. Aspecto que se ha desarrollado previamente, donde están incluidas tanto la comunicación de marketing previa a la crisis, como la que se está llevando a cabo durante la misma. De este contexto es de donde podremos sacar la información para comparar ambos tipos de comunicación.

En la segunda etapa, es donde se elige la muestra a analizar, es decir, en este caso los supermercados que se van a analizar. Carrefour e Hipercor han de ser los principales objetos de comparación ya que son dos de los mayores supermercados de nuestro país, por lo que será interesante comprobar cómo varía su comunicación en momentos de crisis. La elección de ambas cadenas, dejando fuera a su mayor competidor, Mercadona, se basa en que ambas han apostado generalmente por un estilo de publicidad más tradicional, ya que Mercadona no hace publicidad desde los años 90, cuando estableció su modelo de calidad total. Hay algunas excepciones como un anuncio en 2017 con Miguel Induráin, pero su política de marketing es completamente distinta a las marcas elegidas. Además, su nicho de mercado está basado en costes y localización y no en la diferenciación tradicional.

Finalmente, en la tercera etapa se procederá a la comparación de los objetos de estudio con el fin de obtener las diferencias y semejanzas esperadas. Es decir, se comparará la comunicación en redes sociales de los supermercados de estudio antes de la COVID-19 con su comunicación cuando comenzó la crisis de este. El estudio se centrará en analizar los mensajes e ilustraciones lanzadas por ambos supermercados en dicho periodo. Esta fase se desarrollará en el siguiente apartado.

\section{DISCUSIÓN}

A continuación, se analizan los resultados de esta investigación, comparando la comunicación de los supermercados mencionados antes de la COVID-19 y durante el 
mismo. Pero antes mencionar una serie de etapas que han atravesado durante esta crisis, ya no solo negocios como los supermercados si no negocios de toda clase.

En primer lugar, las primeras semanas de confinamiento, en las cuáles el sentimiento de incertidumbre y miedo era evidente en toda la población, las empresas comenzaron a lanzar mensajes de ánimo del tipo "este virus lo paramos juntos" para hacer sentir a la gente que no estaban solos, a pesar del confinamiento evidente. En este primer punto, ambas cadenas trataban de estimular los sentimientos de empatía, solidaridad y unión. El mensaje buscaba activar a los consumidores en una actitud positiva.

En una segunda etapa, en las siguientes semanas de confinamiento, las marcas comenzaron a emitir mensajes con referencia al confinamiento, pero siempre abordando el problema de una manera positiva, mostrando la parte más agradable del mismo, las familias reunidas, el tiempo libre en común, todo aquello que, en la llamada normalidad, es más complicado de llevar a cabo.

La siguiente etapa, aproximadamente en la tercera semana de confinamiento, cuando la gente comienza a preocuparse por aspectos ajenos al virus (desempleo, facturas a pagar, crisis económica...), las empresas comienzan a lanzar mensajes mostrando el valor de las personas detrás de cada producto o servicio, demostrando a la sociedad lo importante que es.

Y la última etapa hasta la fecha se lleva a cabo cuando se comienza a hablar sobre el desconfinamiento, a finales de abril. En esta etapa las empresas comienzan a lanzar mensajes de precaución, y determinados sectores encabezan y aprovechan estos mensajes, como es el de la limpieza, esencial en dicho desconfinamiento.

Uno de los aspectos más curiosos de todo el confinamiento fue la excesiva preocupación por el papel higiénico que fue comprado en cantidades ingentes como si fuese a existir falta de abastecimiento. Sin embargo, todos los supermercados y grandes superficies reaccionaron bien ante la alta e inesperada demanda.

\subsection{Hipercor}

En primer lugar, se puede ver cómo en la comunicación realizada antes de la COVID-19, Hipercor llevaba a cabo sobre todo una comunicación basada en las promociones e información acerca de los productos y servicios de los que dispone. Añadir también que llevaba a cabo acciones de RSC, basadas sobre todo en la donación de comida a bancos de alimentos, así como en su compromiso con el reciclaje. A pesar de estas acciones, aproximadamente 7 de cada 10 publicaciones en sus redes sociales se basaba únicamente en mostrar los productos nuevos de los que disponía, mientras que tan solo 3 de cada 10 iban enfocados a los intereses reales de los clientes, es decir, publicaciones que fueran útiles para éstos, como pueden ser recetas de comida. 
Esa comunicación estaba basada en una política tradicional de comunicación, más centrada en el producto que en el consumidor y su necesidad propiamente dicha. No se tenía demasiado en cuenta cómo podía responder la sensibilidad del cliente, sino que se le informaba continuamente.

En cambio, durante la crisis de la COVID-19, las publicaciones en sus redes dieron un giro radical. Todas sus publicaciones fueron dirigidas al cliente, donde se incluyen más recetas de comida, enfocadas a que la gente se entretenga y probase cosas nuevas, en consonancia con las etapas antes comentadas. También se destacan los mensajes de ánimo, así como las acciones de RSC que están llevando a cabo.

Es decir, desde Hipercor se están enfocando más en transmitir mensajes de apoyo al cliente, dejando de lado las promociones de sus productos, que ahora quedan relegadas a un segundo o tercer plano, dando prioridad al bienestar de sus clientes en estos momentos difíciles.

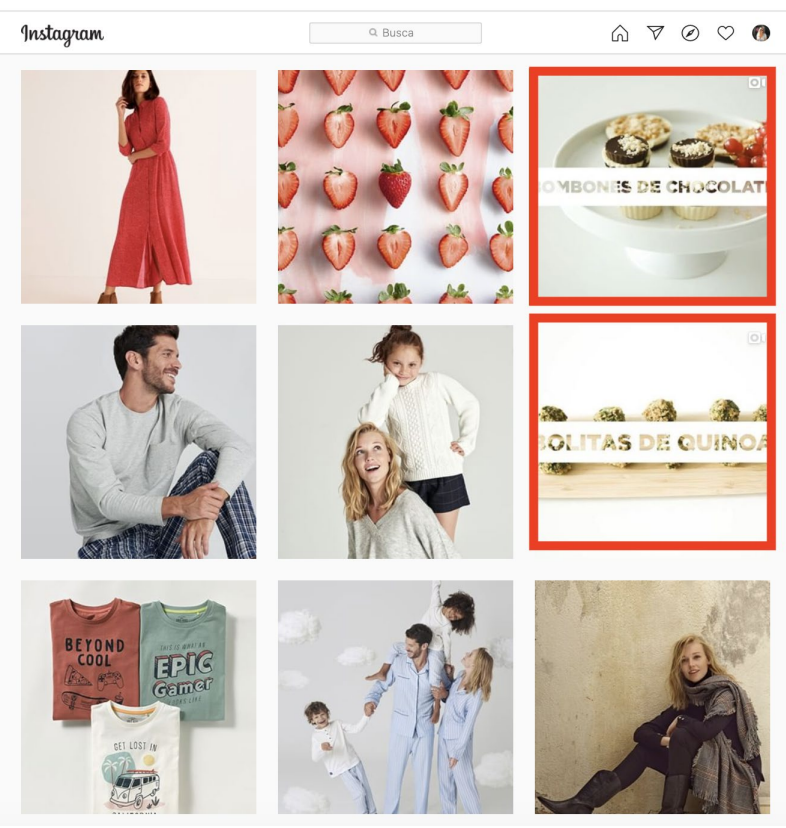

Figura 2: Redes sociales antes de la COVID-19: Mensajes de neuromarketing lanzados por Hipercor.

Fuente: Recopilación por los autores de las redes sociales de la empresa (2020). 


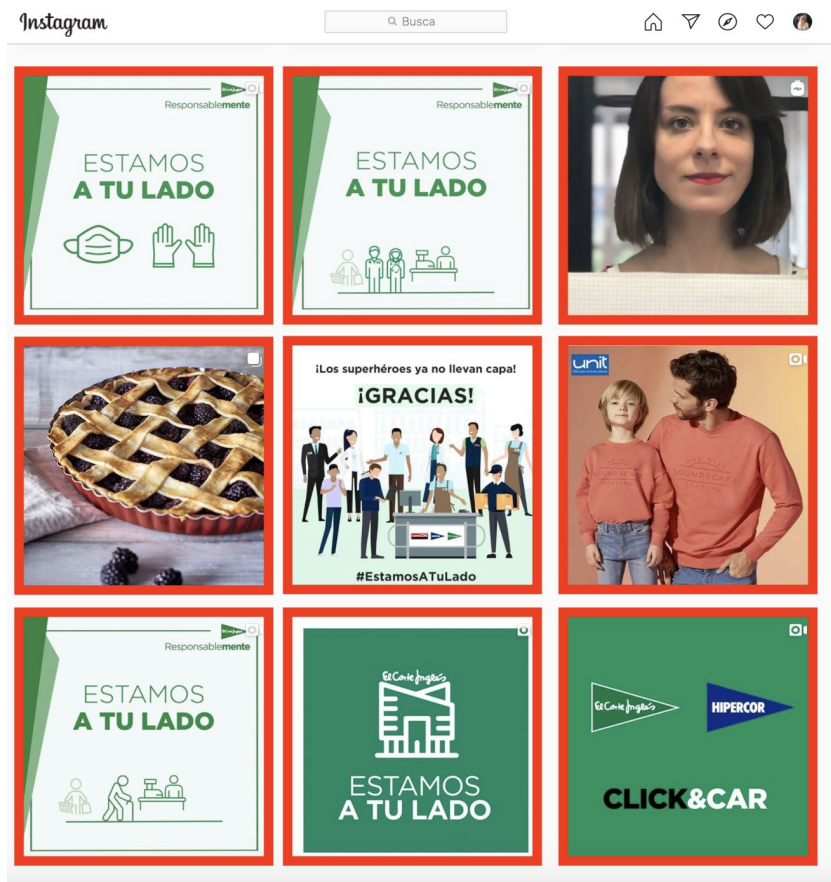

Figura 3: Redes sociales durante de la COVID-19: Mensajes de neuromarketing lanzados por Hipercor.

Fuente: Recopilación por los autores de las redes sociales de la empresa (2020).

\subsection{Carrefour}

En segundo lugar, se puede ver cómo la comunicación de Carrefour realizada antes de la COVID-19 era bastante similar a la realizada por Hipercor, donde predominaba la comunicación de promociones, así como la realización de sorteos, degustaciones y ofertas enfocadas a sus clientes. Hay que añadir también que, en el caso de Carrefour, también realizaba numerosas acciones de RSC para apoyar el mantenimiento medioambiental, el reciclaje o la donación de alimentos a bancos necesitados. En este caso, aproximadamente 9 de cada 10 publicaciones en redes sociales iban destinadas a la promoción de sus productos, dejando tan solo 1 de cada 10 a acciones más enfocadas a los clientes, como la publicación de recetas.

Tras la aparición de la COVID-19, sus publicaciones fueron variando paulatinamente, publicando al principio algún consejo de prevención, y enfocando después todas sus publicaciones al apoyo, ánimo y motivación de sus clientes. Este pequeño goteo de consejos al principio se convirtió en una corriente continua de mensajes positivos y que intentaban buscar una mayor satisfacción y estabilidad mental: recetas para cocinar en casa, vídeos de sus diferentes establecimientos mostrando sus medidas de seguridad y mostrando el buen ambiente que hay entre sus empleados a modo de ser capaces de transmitir esa esperanza que necesitaba la sociedad de que todo saldría bien, y de que ellos estaban trabajando para ello. 
Es decir, una vez más, se muestra que, durante esta crisis, el supermercado ha decidido enfocarse en el cliente, dejando en un segundo plano la promoción de sus productos, haciéndole ver a los clientes que su máxima preocupación recae en ellos.

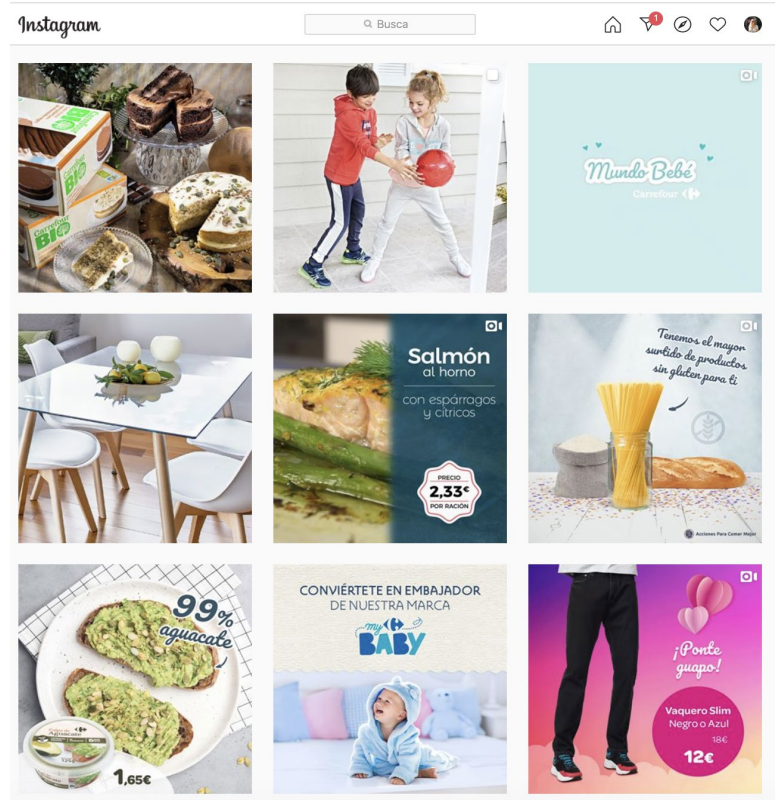

Figura 4: Redes sociales antes de la COVID-19: Mensajes de neuromarketing lanzados por Carrefour.

Fuente: Recopilación por los autores de las redes sociales de la empresa (2020).

Instagram
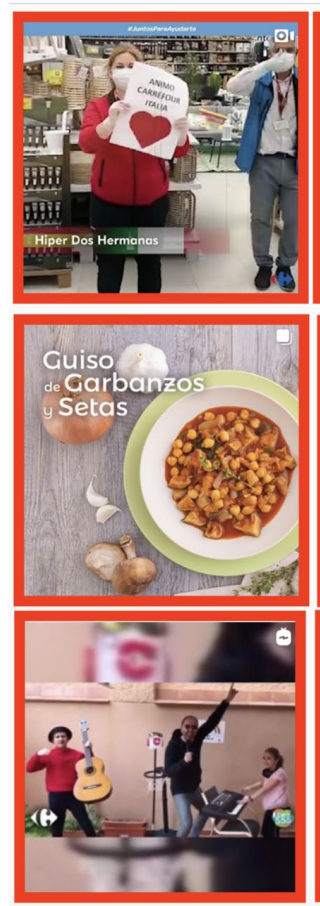

ก
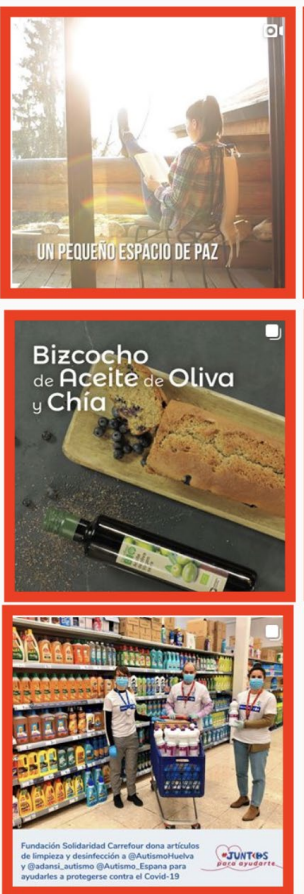
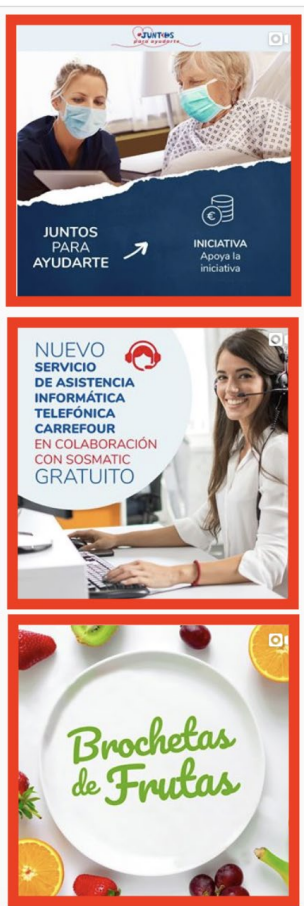

Figura 5: Redes sociales durante de la COVID-19: Mensajes de neuromarketing lanzados por Carrefour. 
Fuente: Recopilación por los autores de las redes sociales de la empresa (2020).

\section{CONCLUSIONES}

Los resultados obtenidos en la comparación de la comunicación realizada por los distintos supermercados antes de la crisis de la COVID-19 y durante la misma, han permitido realizar las siguientes conclusiones acerca del tratamiento del neuromarketing en tiempos de crisis.

Se ha podido observar que la comunicación y sobre todo el mensaje y el contenido de este de los diferentes supermercados varía dependiendo tanto del supermercado como tal, como del momento en el que se encuentren, y más aun tratándose de una crisis mundial como la que se está atravesando en estos momentos. Se ha visto que, en tiempos sin peligro, sin crisis, la comunicación de los diferentes supermercados suele ser puramente comercial, es decir, suele basarse principalmente en la promoción y venta de sus productos y servicios. Sí es cierto que todos ellos, cada uno en la medida que creyera oportuna, llevaba a cabo acciones más enfocadas al cliente, como pueden ser acciones de RSC o acciones de comunicación para acercarse más al cliente (dándoles consejos, recetas, o informándoles de diferentes aspectos de interés para ellos, como las medidas de seguridad que hay que llevar a cabo en estos momentos).

En cambio, en momentos de crisis, dicha comunicación da un cambio radical. Los supermercados buscan apoyar al cliente, lanzarle mensajes de ánimo, mensajes positivos, mensajes que les "humanicen" como empresas, de forma que la gente los vea con otros ojos, que no sientan que lo único que buscan es el aumento puro de los ingresos. Intenta hacerles ver que no están solos y que si todos cooperan esta situación terminará pronto. Es decir, se olvidan de la publicidad en sí misma, la promoción como tal, el mensaje puramente comercial, deja de tener validez en estos tiempos. A la gente le "da igual" el precio de unos macarrones, si no que busca no quedarse sin ellos en estos momentos de miedo e incertidumbre. Es por esto que los supermercados saben en gran medida que ventas van a tener sí o sí, y buscan más acercarse al cliente de una forma más sentimental y menos comercial. Intentan hacerle ver que no piensan en él como un simple número e intentan mostrar esa parte "humana" de los negocios que se mencionaba anteriormente. Es también por esto que los supermercados están realizando más acciones de RSC como donar alimentos o ropa de cama para los hospitales, establecen unos protocolos de seguridad, dando prioridad a las personas más vulnerables, informan a la gente acerca de las medidas de seguridad recomendadas por las organizaciones especializadas, etc.

Desde lo estudiado durante este trabajo, en tiempos de crisis es donde el neuromarketing sale más a la luz. El neuromarketing es una ciencia que estudia detalladamente las respuestas de los clientes y consumidores de una empresa ante un estímulo concreto, para lo que utiliza técnicas de la neurociencia (Kotler y Armstrong, 2012). Es decir, estudia el funcionamiento del cerebro y el 
comportamiento del consumidor. Es por este motivo que los supermercados se están valiendo de esta ciencia para realizar su comunicación, tanto interna como externa, durante la crisis de la COVID-19. El cambio radical del tipo de mensajes emitidos por estos negocios se ha dado debido al estudio del comportamiento del consumidor, y en parte, al estudio de su cerebro. No es difícil pensar que, en esta situación de crisis, la gente está sometida a mucho miedo, estrés e incertidumbre, y que por este mismo motivo no se guían de los mismos impulsos que de habitual, ya que no piensan igual, tienen otros intereses y otras preferencias en su cabeza. Por tanto, los supermercados tampoco pueden emitir los mismos mensajes que antes.

El cliente ha necesitado, durante todo este confinamiento, sentir y esa es la expresión, sentir que los establecimientos cotidianos estaban lanzando mensajes de apoyo constantes e intentaban empatizar con sus miedos y malos momentos.

Además, se ha visto que dependiendo de la "etapa" en la que nos encontremos de dicha crisis, o más concretamente, del confinamiento, las empresas buscan lanzar mensajes diferentes hacia la sociedad, ya que los temas sobre los que se preocupa ésta van cambiando a medida que avanza la crisis. Al principio todo era miedo e incertidumbre, por lo que los mensajes eran más de apoyo y esperanza, "juntos saldremos de esto". Después, las empresas se centraron en mostrar lo bueno del confinamiento, la unidad que formaba, la unión de familias y amigos. La siguiente etapa comenzó a mostrar la preocupación por la crisis prominente que se avecina, por lo que las empresas comenzaron a mostrar la importancia de cada una de las personas en los procesos de producción de productos y servicios, por ejemplo, Carrefour subió varios vídeos a sus redes mostrando a sus diferentes empleados ayudando en el proceso de producción de sus alimentos. Posteriormente, la última etapa hasta el momento muestra la preocupación de la gente respecto al desconfinamiento gradual. La gente tiene miedo de salir, por lo que, las empresas han comenzado a lanzar mensajes en los que muestran las medidas de seguridad que se toman en sus establecimientos (ejemplo las publicaciones en el Instagram de Carrefour).

Ya no solo se habla, por tanto, de los mensajes que lanzan por sus redes sociales únicamente, si no cada una de las acciones que llevan a cabo en sus establecimientos, las medidas de seguridad que están tomando para conseguir que todo el mundo esté lo más protegido posible. Todo esto llega al cerebro de los consumidores y les deja una imagen de la marca que tendrán en cuenta para futuras compras, tanto si lo están haciendo bien como si no, por tanto, estamos sin ninguna duda ante técnicas de neuromarketing. Si una persona ve que una empresa ha tomado todas las medidas de seguridad recomendadas por las autoridades y las ha implementado de manera estricta por el bien de sus consumidores y de sus propios empleados, tanto los unos como los otros aumentarán su fidelidad y confianza hacia la empresa, ya que ésta se ha preocupado por ellos en dichos momentos difíciles. En cambio, si otro supermercado no implementa dichas medidas de seguridad, o las implementa de un modo menos estricto, como es el caso de algunos Día, las respuestas de los consumidores será la contraria, aumento de la desconfianza y disminución de la 
lealtad por dicha marca. Por tanto, se puede ver aquí que el neuromarketing es una técnica que es de utilidad tanto en la comunicación externa de la empresa (clientes), como en la interna (personal).

En definitiva, las técnicas de neuromarketing utilizadas durante una crisis van enfocadas a la humanización de la empresa, la búsqueda de empatía hacia sus clientes, el aumento de la fidelidad por parte de éstos y el aumento de confianza hacia la marca, que en los peores momentos hace todo lo posible por apoyar a los suyos y no dejarles solos ante tal incertidumbre. De forma que todos estos gestos y todas esas acciones sean percibidas por el cliente de manera positiva acercándole un poco más a la marca y reteniéndole.

Para ir concluyendo, en lo que respecta a lo que diferencia al neuromarketing del marketing tradicional, se puede llegar a la conclusión de que es prácticamente lo mismo, ambos buscan comunicarse con los clientes y llegar a ellos de la mejor manera posible, apoyándose en numerosas ocasiones del marketing sensorial o de la RSC para llegar de una manera más eficaz al cerebro de los consumidores, muchas veces sin que ellos mismos se den cuenta. La única diferencia que veo del neuromarketing es que sus estudios se basan más en hechos científicos, a través de técnicas de la neurociencia obtiene respuestas más exactas acerca del comportamiento del consumidor, es decir, es más fácil interpretar los resultados de sus técnicas de trabajo.

\section{REFERENCIAS}

Abuin Vences, N., Díaz Campo, J. y García Rosales, D. F. (2020). Neuromarketing as an Emotional Connection Tool Between Organizations and Audiences in Social Networks. A theorical Review. Frontiers in Psychology, 11:1787. https://doi.org/10.3389/fpsyg.2020.01787

Aguirre, E., Mahr, D., Grewal, D., de Ruyter, K. y Wetzels, M. (2015). Unraveling the personalization paradox: the effect of information collection and trust-building strategies on online advertisement effectiveness. Journal of Retailing, 91(1), 34-49. https://doi.org/10.1016/j.jretai.2014.09.005

Areni, C. S., \& Kim, D. (1994). The influence of in-store lighting on consumers' examination of merchandise in a wine store. International Journal of Research in Marketing, 11(2), 117-125. https:// doi.org/10.1016/0167-8116(94)90023-X .

Berč̌k J., Horská E., Gálová J. \& Sri Margianti E. (2015). Consumer Neuroscience in Practice: The Impact of Store Atmosphere on Consumer Behavior. Periodica Polytechnica Social and Management Sciences, 24(2), 96-101. https://doi.org/10.3311/PPso.8715. 
Braidot, N. (2005). Neuromarketing, Neuroeconomía y Negocios. Editorial Puerto NorteSur.

Cervantes Guzmán, J. N., Vázquez Ávila, G. y Borbolla Elizondo, F. J. (2016). Ventas al cliente final de la PYME comercial de la industria joyera en Guadalajara: Calidad en el servicio y Neuromarketing. Mercados y Negocios, 1 (33). https://dialnet.unirioja.es/servlet/articulo?codigo $=5811254$

Ciprian-Marcel, P., Radomir Lacramioara, R., Maniu, A. I. \& Zaharie M. M. (2009). Neuromarketing - Getting Inside The Customer's Mind. Annals of Faculty of Economics, $4(1)$, 804-807.

https://www.researchgate.net/publication/41163693_Neuromarketing_getting_inside_the_customer $\% 27 \mathrm{~s} \_$mind

Gómez Díaz de León, C., y León de la Garza, E. A. (2015) Método comparativo. En G. Támez González y K. Saénz López (eds.) Métodos y técnicas cualitativas y cuantitativas aplicables a la investigación en ciencias sociales (pp. 223-251). Tirant lo Blanch Humanidades.

Gómez-Yepes, D. (2014). Análisis del impacto de las estrategias de mercadeo en redes sociales en el consumidor mediante técnicas de neuromarketing. Universidad Nacional de

Colombia. https://repositorio.unal.edu.co/bitstream/handle/unal/53766/1053797381.pdf?s equence $=1 \&$ is Allowed $=y$

Hess, E. (1975). Tell Tale Eye: How Your Eyes Reveal Hidden Thoughts and Emotions. Van Nostrand Reinhold.

Horská, E., y Berč̌k, J. (2014) The influence of light on consumer behavior at the food market, Journal of Food Products Marketing, 20(4), 429-440. http:// doi.org/10.1080/10454446.2013.838531.

Klaric, J. (2011). Estamos Ciegos. Planeta Colombia

Koenigstorfer, J., Wasowicz-Kirylo, G., Stysko-Kunkowska, M. y Groeppel-Klein, A. (2013). Behavioural effects of directive cues on front of package nutrition information: The combination matters!. Public Health Nutrition, 8(16), 1-7. http://doi.org/10.1017/S136898001300219X

Kotler, P. y Armstrong, G. (2012). Marketing. (14 ed). (L. Pineda, Trad.) Pearson Educación de México.

Lang, P. J. (1995). The emotion probe. Studies of motivation and attention. The American Psychologist, 50(5), 372-385. https:// doi.org/10.1037/0003-066X.50.5.372 
Lee, E. J. (2016). Empathy can increase customer equity related to pro-social brands. Journal of Business Research, 69(9), 3748-3754. https://doi.org/10.1016/j.jbusres.2015.05.018

Matellanes Lazo, M. (2016) Valoración de las acciones de street marketing en España. Doxa Comunicación, 22, 31-55. https://doi.org/10.31921/doxacom.n22a2

Milosavljevic, M., Navalpakkam, V., Koch, C. y Rangel, A. (2011). Relative visual saliency differences induce sizable bias in consumer choice. Journal of Consumer Psychology, 22 (1), 67-74. https:// doi.org/10.1016/j.jcps.2011.10.002

Muñoz-Leiva, F., Hernández-Méndez, J. y Gómez-Carmona, D. (2018) Measuring advertising effectiveness in Travel 2.0 websites through eye-tracking technology. Physiology E Behaviour 200, 83-95. https://doi.org/10.1016/j.physbeh.2018.03.002

Navarro García, F. (2012). Responsabilidad social corporativa: teoría y práctica. (2da ed.) ESIC Libros profesionales de empresa.

Peattie, K. y Charter, M. (2003). Green marketing. En M.K. Baker (Ed.) The marketing book. pp. 226 - 256. (5ta ed.). Butterworth-Heinemann.

Piqueras-Fiszman, B., Velasco, C., Salgado-Montejo, A. \& Spence, C. (2013). Using combined eye tracking and word association in order to assess novel packaging solutions: A case study involving jam jars. Food Quality and Preference, 28(1), 328338. https:// doi.org/10.1016/j.foodqual.2012.10.006

Plassmann, H., O'Doherty, J., Shiv, B. \& Rangel, A. (2008). Marketing actions can modulate neural representations of experienced pleasantness. Proceedings of the National Academy of Sciences, 105(3), 1050-1054. https://doi.org/10.1073/pnas.0706929105

Pradeep, A. K. (2010). The buying brain secrets for selling to the subconscious mind. Wiley.

Sashi, C. M. (2012). Customer engagement, buyer-seller relationships, and social media. Management Decision, 50(2), 253-272. https://doi.org/10.1108/00251741211203551

Wind, Y. J. (2004). Marketing as an engine of business growth: a cross-functional perspective. Journal of Business Research 58 (7), 863-873. https://doi.org/10.1016/j.jbusres.2004.01.002 


\section{AUTORES}

Patricia Ardanza-Ruiz (1998)

Graduada en Publicidad, Relaciones Públicas y Marketing por el Centro Universitario Cesine de Santander, adscrito a la London Metropolitan University del Reino Unido. En la actualidad, estudia un Curso Superior de Diseño Gráfico y Visual impartido conjuntamente por Deusto Formación y la Universidad Internacional de Valencia. En su periodo como estudiante consiguió dos premios en festivales publicitarios nacionales: el premio al "mejor uso del lenguaje en spot web" en el Publifestival por con un spot social y el premio al "joven talento en mejor originalidad en spot web" en el Smile Festival.

José M. Lavín (1971)

Licenciado en Ciencias Políticas y Sociología por la UNED; Maestro en Cooperación Internacional Unión Europea - América Latina por el Instituto de Investigación Mora (México); Máster Oficial en Ingeniería de la Decisión y Doctor en Investigación para la Comunicación, ambos títulos por la Universidad Rey Juan Carlos En la actualidad, es director del grado de Publicidad, Relaciones Públicas y Marketing por el Centro Universitario Cesine de Santander. Anteriormente, ha sido docente investigador en la Universidad Técnica de Ambato (Ecuador), Universidad Rey Juan Carlos, e investigador en el Centro de Estudios Europeos de la Universidad Nacional Autónoma de México (México). Tiene diversas publicaciones en las indexaciones ELSEVIER, SCOPUS, IEEE, Latindex, etc. Ha dirigido tres tesis de doctorado en la Universidad Rey Juan Carlos, numerosos trabajos de fin de máster y grado y ha participado y dirigido varios proyectos de investigación.

Orcid ID: https:/ / orcid.org/0000-0002-4305-5154

Google ScholaR: https:/ / scholar.google.es/citations?hl=es\&user=RbC7dNcAAAAJ

Scopus: https:// www.scopus.com/authid/detail.uri?authorId=56639282200

Researchgate: https://www.researchgate.net/profile/Jose_Lavin4

Redalyc: https:/ / www.redalyc.org/ autor.oa?id=42292 